Bull. Mater. Sct., Vol. 4, No. 2, April 1982, pp. 133-147. (C) Printed in India

\title{
An experimental study of surface-tension induced convection at reduced gravity
}

\author{
SIMON OSTRACH \\ Case Western Reserve University, Cleveland, Ohio 44106, USA \\ MS received 19 June 1981
}

\begin{abstract}
Fluid motions are induced by surface-tension gradients on the free surface of a liquid in a cylindrical container which is in free fall in a drop tower. Identical experiments are conducted in a normal gravitational environment. The motion in that case is due to natural convection. The ratio of surface-tension to buoyancy forces in the tests is of the order of $10^{4}$ at reduced gravity conditions and 0.13 under normal gravity. In each case a two way flow occurs throughout most of the liquid. Comparison of the results from the two types of experiments is made in order to indicate differences in the flow details.
\end{abstract}

Keywords. Thermocapillary flow; reduced gravity.

\section{Introduction}

This artiole discusses details of an experiment to study the surface-tension induced convection under reduced gravity and to compare it with normal gravity convection with all other conditions identical. The intensities of such flows and their penetration depth below the free surface are also determined. In this way the dominant role of a free surface in a reduced gravity environment is indicated.

In two earlier papers by the author in these proceedings, ane on the convection phenomena at reduced gravity and the other on motion in duced by capillarity, the convection induced by surface or interfacial tension gradients has been discussed in detail. These gradients, which can arise due to gradients in temperature or concentration at the surface or irterface, can generate conventional convection flows or unitable cellular flows just as gravity induces these two types of natural convection flows in the presence of density difference. When caused by temperature gradients, the dimensionless parameters related to gravitational convection is the Grashof number, $\mathrm{Gr}$, and that corresponding to surface-tension induced convection is the Marangoni number, Ma. It is noted that the Marangani number is independent of gravity and the Grashof number is independent of surface tension. Thus, the relative magnitude of surface tension and buayancy forces is given by the ratio $\mathrm{Ma} / \mathrm{Gr}=v(\partial \sigma / \partial T) / \rho \beta g L^{2} k$. The quantities appearing in the ralation have been defined in the earlier papers. The experiment being discussed here was designed so that this ratio $\mathrm{Ma} / \mathrm{Gr}$ takes on valucs from about 0.13 to $1.5 \times 10^{4}$ in normal and reduced gravity environments, respectively.

Many possible modes of flow and configurations of interest in which the surface tension can be significant have also been discussed earlier, in particular the role 
of free surface on reduced gravity convection. The latter is also of particular interest in the present experiment.

Amang the existing publications related to this problem are the ones reported in Babskiy et al (1973), Birikh (1966), Levich (1962) and Yih (1968). Babskiy et al (1973) consider the steady flow generated by the free surface in a rectangular enclosure in which the two opposite walls that bound the free surface are at different temperatures. It is indicated that the solutions are valid for sufficiently small Marangoni numbers $(\sim 1 \cdot 0)$ yet results are presented for values of the parameters of 5 and 500. An exact solution is found by Birikh (1966) for the equations for free convection and surface tension in an infinite plane-parallel horizontal layer of liquid with a constant temperature gradient in the horizontal direction. Here also the steady state has been considered. Levich (1962) has discussed purely capillary convection. Yih (1968) has pointed out many inconsistencies in his solution and has given a corrected solution of the Levich problem.

The only reduced gravity experiments on surface-tension driven flows are those induced by a thermal instability (Grodzka and Bannister 1972) (heating from below) and, thus, are different from the configuration of interest herein. Since there did nat appear to be any experimental study of conventional convection due to surface tension alone, the present experiments were developed to be conducted in the NASA Lewis Reser.rch Centre's 500-ft drop tower in which approximately $5.5 \mathrm{sec}$ of reduced gravity (about $10^{-5} \mathrm{~g}_{0}$ ) can be obtained. From an order of magnitude analysis (Pradhan and Ostrach 1976) it was estimated that it would take somewhat less than $3 \mathrm{sec}$ for the surface boundary layer to reach steady state. Thus, worthwhile data could be obtained in that facility.

\section{Experimental set-np}

The drop tower facility imposed a number of constraints on the test apparatus. The size is limited by that of the drop module and the instrumentation and power supply have to conform to the capsule capacity. In addition, it must be able to withstand the impact of a 40-g deceleration. The test apparatus mounted in a plexiglass leakproof chamber that is maintained at a pressure of 1 a.tm, even when the drop-tower pressure is $100 \mu$ of mercury, is shown in figure 1. The liquid container is a cylindrical plexiglass dish with a $10 \mathrm{~cm}$ i.d. and $3 \mathrm{~mm}$ thick vertical walls of $3.16 \mathrm{om}$ height. The inner wall of the dish is covered with a teflon tape to yield a $90^{\circ}$ contzet angle with distilled water at $25^{\circ} \mathrm{C}$. Considerable preliminary testing (Pradhan and Ostrach 1976) was required to determine this method of maintaining a flat interface and preventing creeping of liquid up the walls at reduced gravity.

Distilled water is chosen as the test fluid because it has the following properties : (a) large surface-tension variation with temperature; (b) low viscosity; (c) small density variation with temperature ; (d) transparency ; and (e) easy accessibility. The temperature gradient is imposed by an infrarcd heat source located above the free surface (see figure 1).

The heat source is positioned vertically above the liquid at the centre of the dish with its bottom $2.5 \mathrm{~mm} \pm 0.05 \mathrm{~mm}$ above the free surface. With such a heating from abave configuration, convection due to buayancy is minimized 


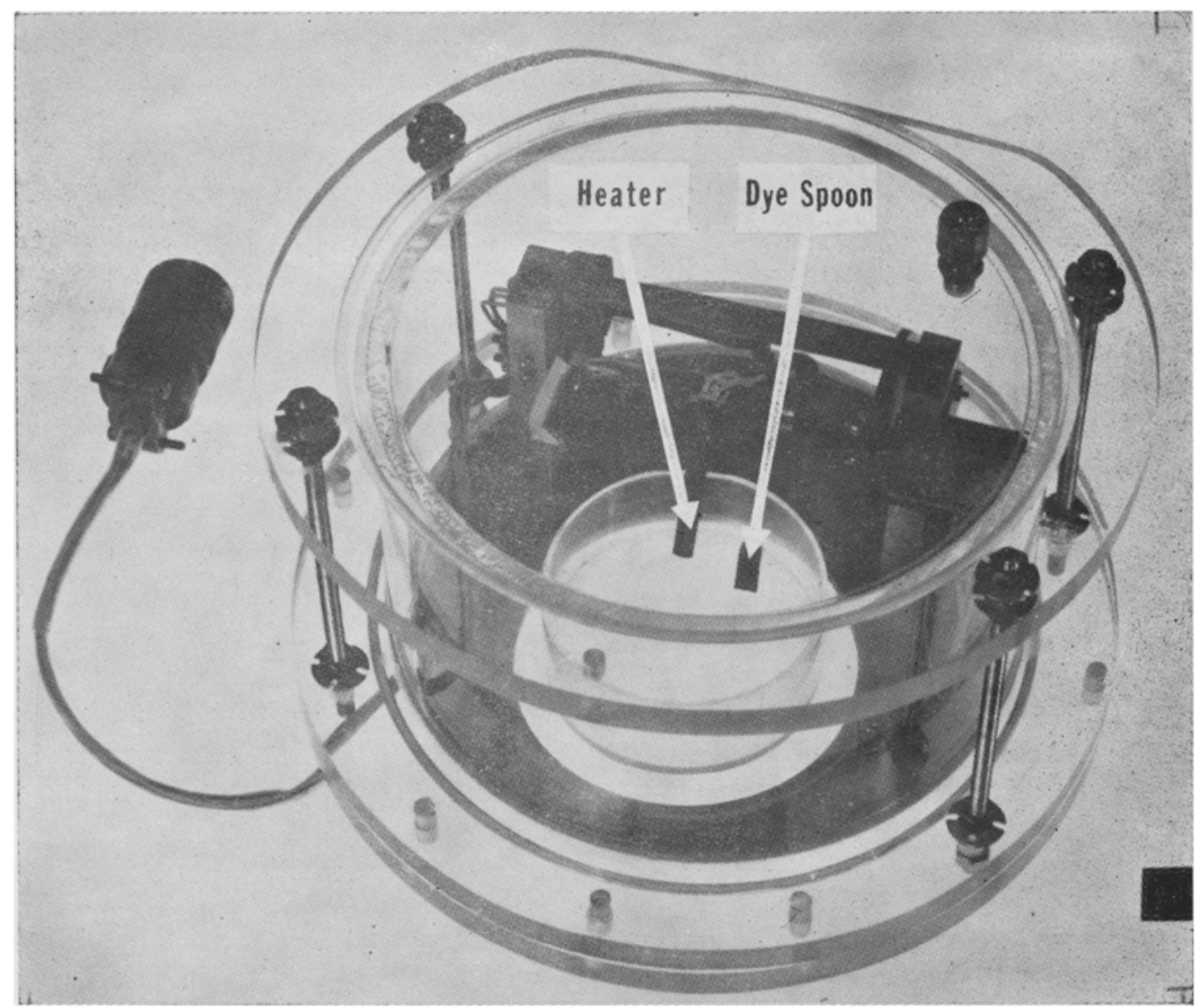

Figure 1. Test container. 
and the temperature gradient on the free surface is impased faster than by heating the side wall.

Methylene blue dye is used to make the flow patterns visible. Four 40-mesh dye particles and $\frac{1}{2} \mathrm{mg}$ of fine ground (400-mesh) dye are placed on top of a knu rled spoon which, when vibrated by means of a $24-\mathrm{V}$ d.c. motor, sprinkles the dye on the liquid free surface about $10 \mathrm{sec}$ before the start of the test. Some or the finely ground dye spreads over the free surface and larger dye particles penetrate into the liquid and form vertical strands. When the fluid is completely stationary and there are no temperature gradients, the dye strands maintain their position and do not diffuse for about a minute. When the test starts and the liquid moves, the dye strands also move and thereby indicate the velacity profiles. Top and side views of the dish are recorded on a movie film by two $16 \mathrm{~mm}$ high speed Melikon cameras.

To measure the radial surface temperature gradient imposcd by the heat source, five thermistor probes (accuracy $\pm 1^{\circ} \mathrm{C}$ ) are placed so that their beads just touch the free surface. To evoid any disturbance on the free surface due to other than $90^{\circ}$ contact angle between probes and water, a shrinkable teflon tube is slipped over the probes.

Some disturbances due to thermistor probes however remain. To correct for these, some velocity data are taken without the thermistors in place. The previous temperature profile readings are considered to bc applicable for these tests as well. The temperature measurements are made once the waves subsided about 1 sec after the release of the module in the drop tower.

Two electronic clocks, which are visible in the films, are used to record time. Further details of the experimental set-up can be seen in Ostrach and Pradhan (1978).

\section{Experimental procedure}

The dish is first cleaned by a special process (Pradhan and Ostrach 1976) to eliminate any possible contamination. The inner wall of the dish is then covered with a single layer of transparent teflon tape. The dish is cleaned again with methyl alcohol and distilled water. The dish is then taped into place. The distilled water is added up to the thermistor beads. The heater is adjusted so that when it comes to position, its bottom is $2.5 \mathrm{~mm}$ from the free surface.

The particles of methylene blue are carefully placed on the vibrator spoon. The test apparatus is then placed in the leak-proof tank which was hydrostatically tested for leaks and found to be good u.p to a p: essu re difference of 13 psi, which is considered sufficient for these tests. The entire apparatus is then assembled in the capsule for the drop-tower test. The drop module is hung in the drop tawer for 45 min so that the fluid can become staticnary before the test.

The following tests are run in the drop tower:

(i) The heat source starts heating the free surface $1 \mathrm{sec}$ after the package is released.

(ii) The free surface is preheated for 10 sec before the drop.

(iii) The free surface is preheated for $40 \mathrm{sec}$ before the drop. 
Test (i) is performed to see how the flow starts with surface tension gradients being dominant. This might also give some idea as to what kind of steady-state flow pattern can be expected for high reciprocal modified Bond number (greater than $10^{4}$ ) cases. Tests (ii) and (iii) show any abrupt changes in flows as the gravity level is drapped to $10^{-5} g_{0}$. All of the tests are performed at normal gravity also, with all initial conditions identical.

Test conditions are : initial temperature, $23^{\circ} \mathrm{C}$, surface-tension temperature, $d \sigma / d T=0.16 \mathrm{dyn} / \mathrm{cm} /{ }^{\circ} \mathrm{C}$, characteristic length of $4.5 \mathrm{~cm}$. The heater is initially heated for about $2 \mathrm{~min}, 35 \mathrm{sec}$ far from the free surface. In this time interval the steady state of the heater is achieved, and it is in this state that the heater is used to heat the free surface. This steady-state time of the heater is measured by means of a thermocouple attached to its lip. Once the calibrations are over, the thermacouple is removed and the heater is heated in all experiments for the same time period ( $2 \mathrm{~min}, 35 \mathrm{sec}$ ). The relevant dimensionless parameters are indicated in table 1 .

\section{Results}

The results of the various experiments are presented in the order in which they were desoribed previously.

Test (i) : The heat source starts heating the free surface 1 sec after the package is released. This 1 -sec time interval is given to eliminate any small disturbances that are produced by the release mechanism. A previous test under identical conditions has shown that the disturbance caused by release is very small and lasts for about $0.5 \mathrm{sec}$.

The surface tension gradient induced mation starts soon after the heater starts to heat the surface as can be seen from the movie frames. The flow starts from the centre clase to the heater and spreads outward as the temperature gradient is established (see figure 2).. The temperature profiles in figure 2 are for a single run and are drawn assuming that the free surface temperature close to the side wall remains the same as the initial liquid temperature in the time interval of 5 sec. This is done to get some idea of the characteristic temperature difference. The temperature profiles in figure 2 are for a test where the beating starts with the release of the package in the drop tower.

Loaking at the movie films, it appears that a very thin film an the surface is pulled away from the heater at considerable speeds because of the induced surfacetension gradient. Very little or no motion could be observed around $3 \mathrm{~cm}$ away from the centre of the dish, where the surface tension gradient is also found to be small. The free surface velocities are shown in figure 3 and represent the velocities measured by tracking the dye particles on the surface, for small velocities like $2 \mathrm{~mm} / \mathrm{sec}$, the dye particle displacement is measured at intervals of $1 \mathrm{sec}$. Thus the velocities shown in figure 3 are those measured from a time interval of 2 to 3 sec after heating.

As soon as heating starts, the dye movement is observed around the heater, but after $2 \mathrm{sec}$ of heating all the free surface is not active. The dye movement is observed only up to a radius of about $2 \mathrm{~cm}$ from the centre because temperature gradients are established in that region only. However, after 3 sec of heating 
Surface-tension inducea convection at reduced gravity

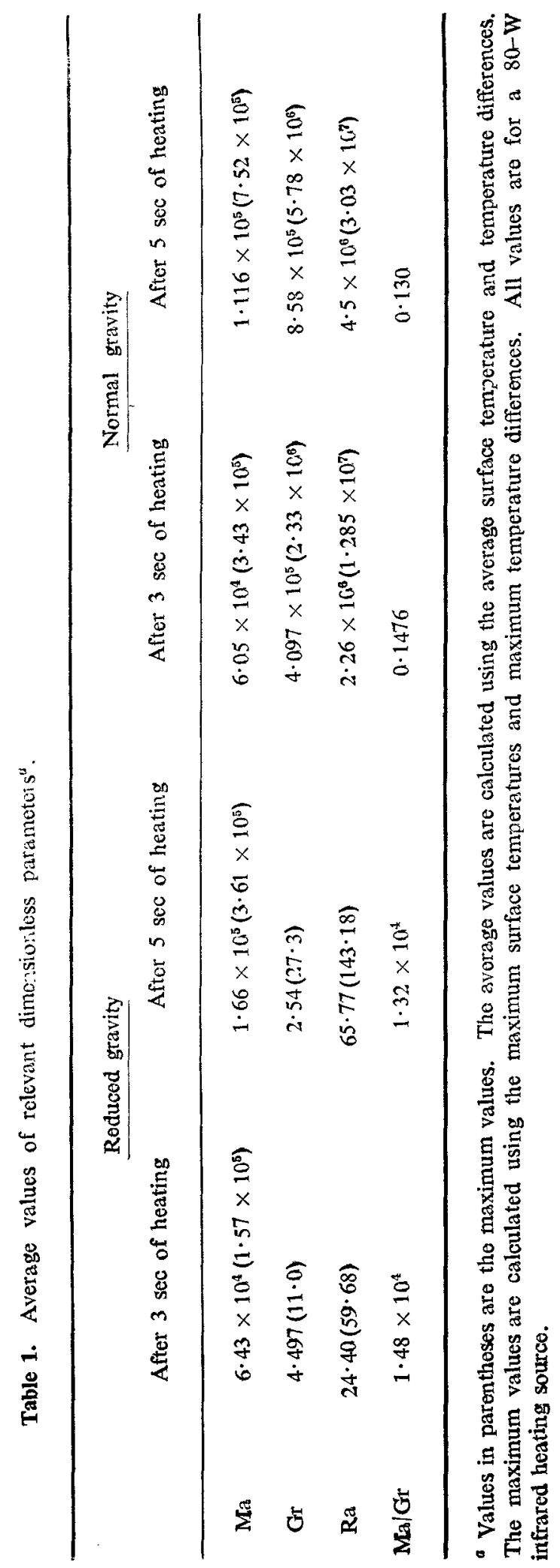


almost all of the dye has moved out from the centre and no measurements can be made there. Velocities measured about $2.5 \mathrm{~cm}$ away from the centre are seen to be the same as those shown in figure 3 .

In the interior of the liquid a two-way motion is observed, i.e., flow away from the heater clase to the free surface and the rest of the fluid moving in the reverse direction ber ause of continuity. Dye strand displacements with time as determined from the films are given in figure 4 . Before the heat is imposed the dye strands are stationary, although they are not necessarily straight and vertical. If vertical velocities are assumed to be negligible, the dye strand displacements can be used to calculate internal fluid velocities. From these figures it can be seen that a boundary layer forms close to the free surface. Outside this free-surface boundary layer the fluid moves toward the centre with a velocity of $1 \cdot 0 \pm 0 \cdot 2$ $\mathrm{mm} / \mathrm{sec}$.

The $(\mathrm{Ma} / \mathrm{Gr})_{\text {arg }}$ ratio is found to be around $10^{4}$ (see table 1) for a given test. The ratio is a function of average liquid temperature and the gravity level. During a specific test its value daes not change to. much (max $10 \%$ ) as the average liquid temperature changes by no more than $10^{\circ} \mathrm{F}$ in the 5 -sec time period. The initial liquid temperature being about $80^{\circ} \mathrm{F}$, this change in liquid temperature gives rise to only small changes in the constants which form $(\mathrm{Ma} / \mathrm{Gr})_{\mathrm{avg}}$ ratio. The average liquid temperature at different time intervals is calculated using the surface temperature profiles given in figure 2 and making use of the following formula

$$
T_{\text {arg }}=\frac{2}{R^{2}} \int_{0}^{R} T(r) r d r,
$$

where $R$ is the dish radius.

The average temperature difference used in the calculation of Marangoni, Grashof, and Rayleigh numbers is as follows :

$$
(\triangle T)_{\text {arg }}=T_{\text {arg }}-T_{w} \text {. }
$$

From the values of dimensionless parameters that are given in table 1 we see that the Marangoni number which represents surface-tension gradient-induced flow is about $10^{4}$ times larger under reduced gravity than the Grashof number which represents buoyancy-induced flow. This indicates that the flow due to surfacetension gradient is dominant.

As the heating is continued we see that the value of $(\mathrm{Ma} / \mathrm{Gr})_{\text {arg }}$ ratio reduced from $1.48 \times 10^{4}$ (at $3 \mathrm{sec}$ ) to $1.32 \times 10^{4}$ (at $5 \mathrm{sec}$ after heating) as the average surface temperature increases. Thou gh the change in the value of $(\mathrm{Ma} / \mathrm{Gr})_{\mathrm{Brg}}$ is small, it shows the trend that the bu oyancy effects keep increasing as heat continue but in the $5 \mathrm{sec}$ of drop time they are still negligible.

The average Marangoni number is found to increase with time-6.43 $\times 10^{4}$ (at $3 \mathrm{sec}$ ) to $1.66 \times 10^{5}$ (at $5 \mathrm{sec}$ after heating)-which mecns the free surface boundary layer does not reach steady state in $5 \mathrm{sec}$ of reduced gravity period and the How is still developing. The free surface boundary layer steady state could not be achieved in $2.81 \mathrm{sec}$, as estimated from an order-of-magnitude analysis because of the reverse flow created by the side wall, which was not considered in the order-of-magnitude analysis. Moreover, in the order-ofmagnitude analysis the effect of heat transfer though the boundaries and vapori - 
Surfacertension induced convertion at reched gravity

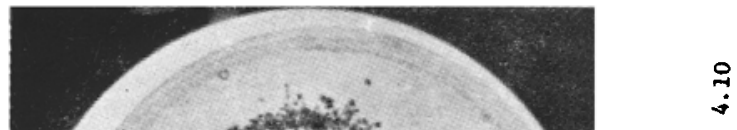

$\stackrel{7}{;}$

คุ

$\stackrel{8}{\dot{0}}$

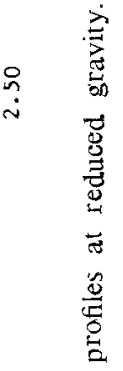

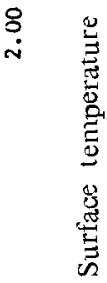

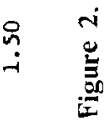

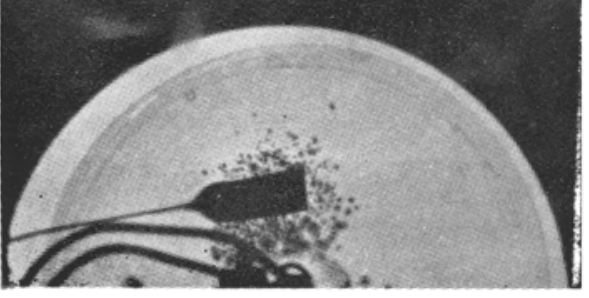

$\stackrel{n}{\mathfrak{i}}$ 


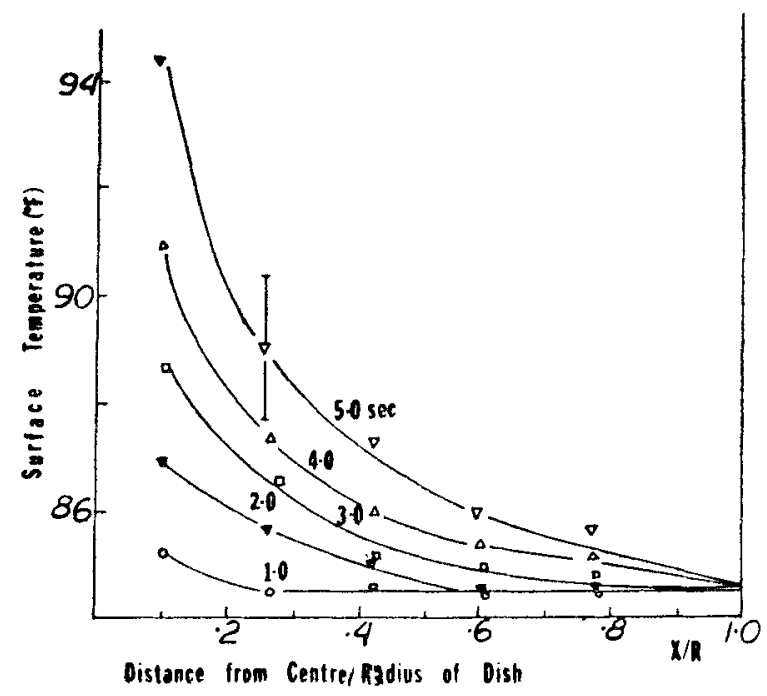

Figure 3. Surface velocity $v s$ distance from centre of dish. Taken $2 \mathrm{sec}$ after heating.

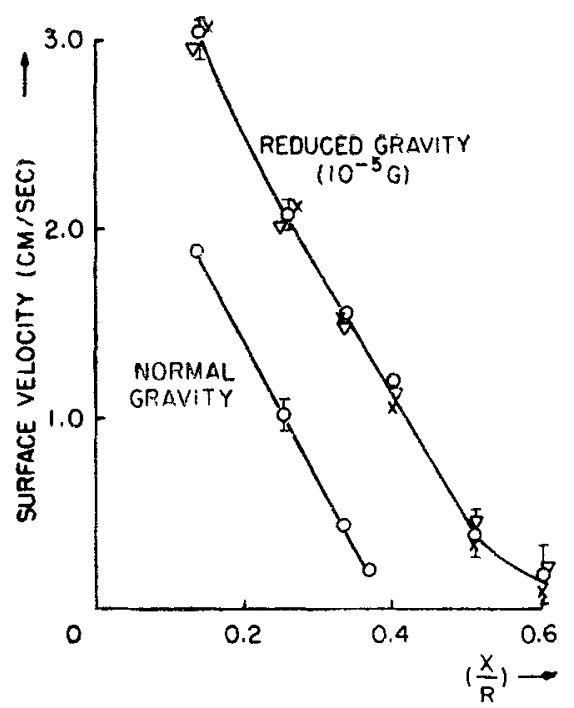

DISTANCE from the CENTER/RADIUS of DISH

Figure 4. Dye displacement with time-reduced gravity.

zation of liquid from the free surface are not considered though they are present in reality and affect the occurrence of steady state.

The two-way motion in the interiar of the liquid and the decrease in surface velocities away from the heater can be explained as follows. The two-way motion in the interior of the liquid can be easily explained by means of continuity. If we consider that the free surface remains close to horizontal with time, as is observed 
in the tcsts, for mass conservation a given mass flow in one direction has to be compensated for by an equal mass flow in the opposite direction far a contained fluid. In order to understand the decrease in surface velocity with radius, we next look at the fluid element inside the free surface. Let us consider a fluid element of $r$ thickness, at a distance $r$ from the centre and located between the free surface and the zero horizontal velocity region as shown in figure 5 . The zero horizontal velooity region is created by the appearance of the two-way flow as mentioned previously. The depth of the zero velacity region from the free surface is $L_{r}$ and $L_{r}+\Delta_{r}$ and the free surface velocities are $U_{r}$ and $U_{r}+\Delta_{r}$, at a distance of $r$ and $r+\Delta_{r}$, from the centre, respectively.

Assuming small vertical velocities in the region, as has bcen found in the order-of-magnitude analysis, we obtain a relation between $U_{r}+\Delta_{r}$ using mass continuity,

$$
\begin{aligned}
& 2 \pi\left(U_{r}\right)\left(L_{r}\right)=2 \pi\left(r+\triangle_{r}\right)\left(U+\triangle_{r}\right)\left(L_{r}+\triangle_{r}\right), \\
& U_{r}+\triangle_{r}=\left(\frac{r}{r+\triangle_{r}}\right)\left(\frac{L_{r}}{L_{r}+\triangle_{r}}\right) U_{r} .
\end{aligned}
$$

It is observed from the experiments that the distance of the zero. horizontal velocity region from the free surface increases as one goes away from the centre because of the diffusion of momentum from the free surface to the interior of the liquid. So, $L_{r}+\Delta_{r}>L_{r}$ for all $r$. Therefore, $U_{r}+\Delta_{r}<U_{r}$. This shows that the continuity itself would lead to a decrease in surface velacity with radius, as is observed in figure 4.

Test (ii): The free surface is preheated for 10 sec before the drop. In the first $10 \mathrm{sec}$ during which the free surface is preheated at normal gravity, the surface motion away from the centre is slower than that explected under reduced gravity conditions. As the experimental package enters the reduced gravity environment the surface velocities are seen to increase.

Comparing the temperature profiles in figures 3 and 9 we see that the temperature difference between the given points and at a given time are larger under normal gravity than in reduced gravity. This means that more heat is being transferred by convection under reduced gravity than in normal gravity because bath

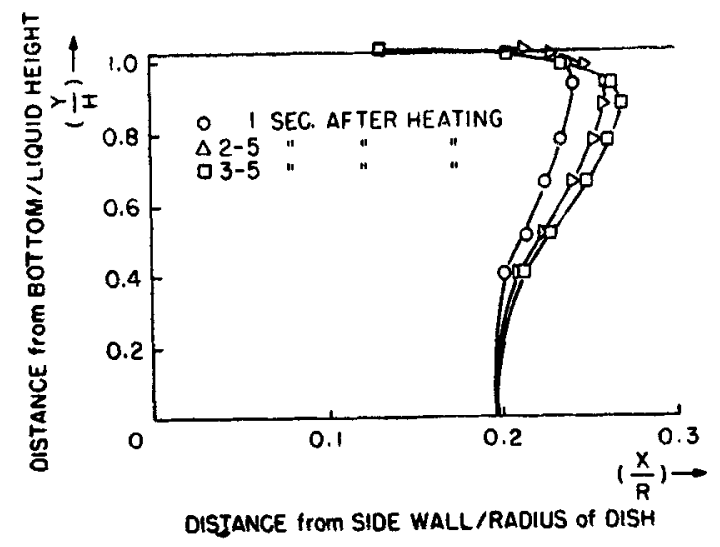

Figure 5. Schematic of fluid element inside the free surface. 
of the tests are performed under identical initial conditions. This supports the aforementioned observation of higher surface velocitics under rcduccd gravity.

Test (iii) : The free surface is preheated for $40 \mathrm{sec}$ before the drop so that a steady state thermal boundary layer seems to be established during this preheat time under normal gravity environment. Except for the free surface effect, such as is abserved in the preceding experiment (test ii), no change in the flow pattern is observed during the $5 \mathrm{sec}$ of reduced gravity. The two-way flow in the intericr of the liquid remains the same.

The three experiments just describcd are repeated for identical initial condition in a normal gravity environment. Dye displaccment curves at different time intervals are given in figures 6 ard 7 . The temperature profilss for this case are presented in figure 8 . The values of relevant dimensionless parameters are given in table 1 .

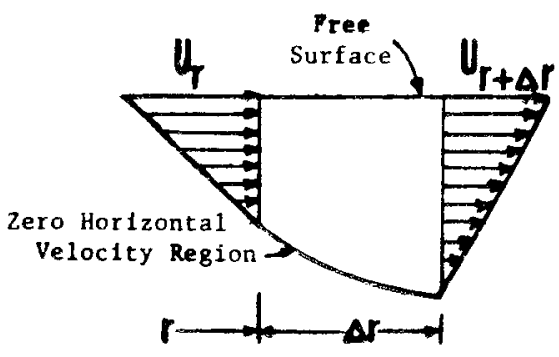

Figure 6. Dye displacement with time-normal gravity.

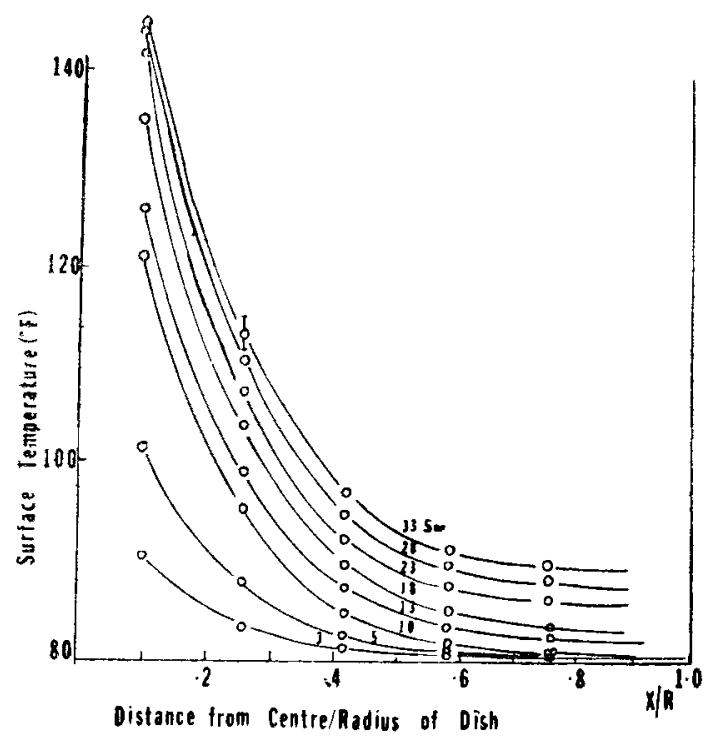

Figure 7. Dye displacement with time-normal gravity. 
The temperature profiles up to $10 \mathrm{sec}$ after heating are drawn assuming the side wall temprature unchanged due to heating and are used to compare the results with the reduced gravity temperature profiles in figure 2. The temperature profiles between $10 \mathrm{sec}$ and $33 \mathrm{sec}$ after heating are used to get some idea of the temperature gradients present when the experimental package is dropped as in tests (ii) and (iii).

Comparison of the results (see figure 3) indicates that surface velocities are considerably higher under reduced gravity conditions than in normal gravity for the same initial conditions. In figure 3 the normal gravity curve is drawn from a single run by traoking the dye particles as they move on the free surface. The velocities are calculated starting at 2 sec after heating in a manner similar to the ones in the reduced gravity test (i). The velocities calculated between 2 and $4 \mathrm{sec}$ seem to remain the same.

It is observed that in the interior of the liquid the boundary layer near the free surface is thicker at $1 \mathrm{~g}$ than at reduced gravity. At normal gravity the Grashof numbers are found to be an order of magnitude larger than the Marangoni

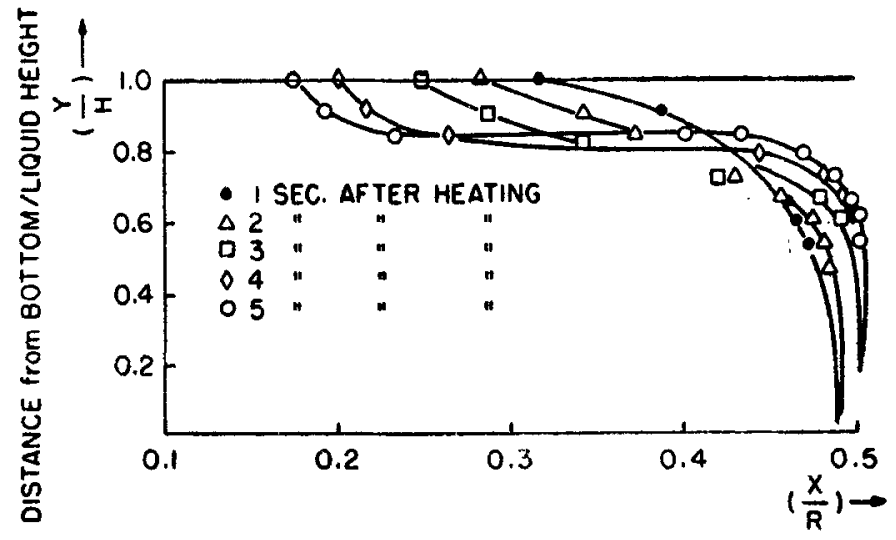

OISTANCE from SIDE WALL/RADIUS of DISH

Figure 8. Surface temperature profiles-normal gravity.

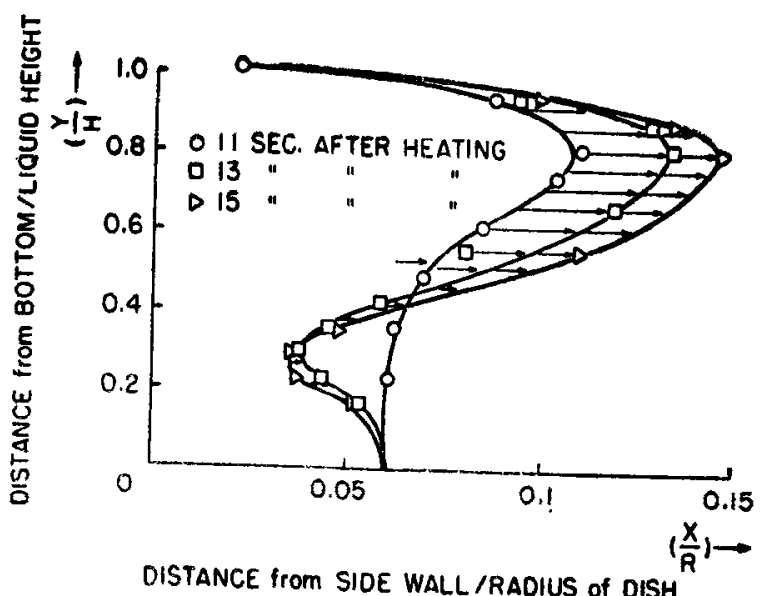

Figare 9. 
numbers, and so the flow due tc density differences and buoyancy is dominant. This gives rise to large internal flows close to the free surface and directed away from the centre of the dish. This flow is large close to the free surface because that is where the tomperature gradients are present. But as heating continues, the heat diffuses downward and increases the Grashof number (see table 1) and ultimately the mass flow, boundary-layer thickness and internal velocities.

Thus we see that though the free surface velocities are smaller the overall mass flow rate away from the centre is quite large due to the large density gradient induced flow. This gives rise to large reverse flow due to continuity requirements.

Although none of the existing work (Babskiy et al 1973; Birikh 1966; Levich 1962 ; Yih 1968) deals with the identical configuration studied herein in that either the configuration or thermal boundary conditions are different and also it all deals with steady flows, the flow profiles (figures 4,6 and 7) are qualitatively similar.

\section{Conclusions}

The present experiments have indicated that surface tension gradients at reduced gravity can be dominant and can generate flows as large as buoyancy at normal gravity.

Under normal gravity the free surface can be said to be passive with respect to the heat-transfer process in the sense that the fluid motion is induced by buoyancy. The buoyancy effects are dominat in the form of a thicker free surface boundary layer produced by larger flow due to density gradients. Moreover, the free surface velacities at normal gravity are smaller than those under reduced gravity for the identical initial conditions.

As the gravity level is reduced $\left(10^{-5} \mathrm{~g}_{0}\right)$ the surface tension gradient farce becomes dominant ( $\mathrm{Ma} \sim 10^{5}$ and $\mathrm{Gr} \simeq 20$ ) and there is negligible gravity-induced convection. In this case, the free surface plays an active role in the heat-transfer process. Surface-tension gradient driven convection is seen to produce a thin boundary layer $(\sim 3 \mathrm{~mm})$ near the free surface within a period of 5 sec and a two-way flow is observed inside the liquid. Free surface velocities as high as $3 \mathrm{~cm} / \mathrm{sec} \pm 0.1 \mathrm{~cm} / \mathrm{sec}$ and internal velacities of the order of $1 \mathrm{~mm} / \mathrm{sec} \pm 0.2$ $\mathrm{mm} / \mathrm{sec}$ in the reverse flow are observed. The foregoing velocities are obtained for the reduced gravity case with an $80-\mathrm{W}$ heater.

\section{References}

Birikh R V 1966 J. Appl. Mech. Tech. Phys. 743

Babskiy V G, Sklovsleayer I L and Sklovskiy Y O 1973 Space studies in Ukraine, No. 1 Space materials studies and technology.

Grodzka P G and Bannister T C 1972 Science 176506

Levich V G 1962 Physiochemical hydrodynamics (New Jersey: Prentice Hall)

Ostrach S and Pradhan A 1978 AIAA J. 16419

Pradhan A and Ostrach S 1976 Surface-tension gradient induced convection under reduced gravity conditions, Case Western Reserve University, Cleveland, Ohio, FTAS/TR-76-123

Yih C S 1968 Phys. Fluids 11447 